\title{
Evaluación del progreso de aprendizaje en lectura dentro de un Modelo de Respuesta a la Intervención (RtI) en la Comunidad Autónoma de Canarias
}

\section{Evaluating progress in reading acquisition within a Response to Instruction model in the Canary Islands}

\author{
Juan E. Jiménez ${ }^{1}$, Doris Luft Baker ${ }^{2}$, Cristina Rodríguez ${ }^{1}$, Patricia Crespo ${ }^{2}$, \\ Ceferino Artiles ${ }^{3}$, Miguel Alfonso ${ }^{3}$, Desirée González ${ }^{1}$, Christian Peake ${ }^{1}$, Natalia Suárez ${ }^{1}$ \\ ${ }^{1}$ Facultad de Psicología, Universidad de La Laguna, Islas Canarias, España. ${ }^{2}$ Center for Teaching and Learning (CTL), University \\ of Oregon, USA. ${ }^{3}$ Dirección General de Ordenación, Innovación y Promoción Educativa de la Consejería de Educación, Universi- \\ dades y Sostenibilidad del Gobierno de Canarias, España.
}

Disponible online 31 de agosto de 2011

\begin{abstract}
El objetivo principal de este trabajo es dar a conocer el sistema de evaluación del progreso de aprendizaje en lectura dentro de un modelo de Respuesta a la Intervención - Nivel 2 de intervención que se viene utilizando en la Comunidad Autónoma de Canarias en el marco institucional de cooperación científica entre el grupo de investigación de la Universidad de La Laguna "Dificultades de aprendizaje, psicolingüística y nuevas tecnologías", el Center on Teaching and Learning de la Universidad de Oregon y la Consejería de Educación, Universidades y Sostenibilidad del Gobierno de Canarias. Dentro de este contexto se viene utilizando el sistema de evaluación Indicadores Dinámicos del Éxito en la Lectura, versión española del Dynamic Indicators of Basic Early Literacy Skills. Su principal finalidad es evaluar el progreso de aprendizaje de la población escolar canaria identificada con "riesgo" de presentar dificultades específicas de aprendizaje en lectura y que participa en un Modelo de Respuesta a la Intervención.
\end{abstract}

Palabras clave: Identificación Temprana; Indicadores Dinámicos del Éxito Lector; Evaluación; Riesgo de Dificultad de Aprendizaje; Dislexia; Fluidez; Modelo de Respuesta a la Intervención.

The main aim of this study is to provide an overview of monitoring progress within a Response to Intervention model (Tier-2, small group intervention) used in the Canary Islands. This programme is conducted within the framework of a scientific cooperation agreement between the University of La Laguna research team "Dificultades de aprendizaje, psicolingüística y nuevas tecnologías" ("Problems in learning, psycholinguistics, and the new technologies"), the University of Oregon's Center on Teaching and Learning, and the Consejería de Educación, Universidades y Sostenibilidad of the Canary Islands Government. The Indicadores Dinámicos del Éxito Lector is used for monitoring progress. The Indicadores Dinámicos del Éxito Lector is the Spanish version of Dynamic Indicators of Basic Early Literacy Skills. Its main purpose is to evaluate reading progress in students identified as "at risk" of developing reading difficulties using an Response to Intervention model.

Keywords: Early Identification; IDEL; Assessment; At Risk Readers; Dyslexia; Fluency; Response to Intervention Model.

Correspondencia: Juan E. Jiménez. Facultad de Psicología. Universidad de La Laguna. Tenerife. (España). E-mail: ejimenez@ull.es. E-mail de los otros autores: Doris Luft Baker: dbaker@uoregon.edu; Cristina Rodríguez: crodri@ull.es; Patricia Crespo: pcrespo@ull.es; Ceferino Artiles: Ceferino.artileshernandez@gobiernodecanarias.org; Miguel Alfonso: mafocab@gobiernodecanarias.org; Desirée González: degonmar@ull.es; Christian Peake: cpeakem@ull.es; Natalia Suárez: nsuarez@ull.es 
El presente trabajo describe el Modelo de Respuesta a la Intervención (RtI) en el nivel 2 (Tier-2) que se viene implementando en la Comunidad Autónoma de Canarias en el marco institucional de cooperación científica entre el grupo de investigación de la Universidad de La Laguna, "Dificultades de aprendizaje, psicolingüística y nuevas tecnologías", el Center on Teaching and Learning (CTL) de la Universidad de Oregon y la Consejería de Educación, Universidades y Sostenibilidad del Gobierno de Canarias. Dentro de este contexto se viene utilizando el sistema de evaluación Indicadores Dinámicos del Éxito en la Lectura (IDEL; Good, Baker, Knutson y Watson, 2006) versión española del Dynamic Indicators of Basic Early Literacy Skills (DIBELS, Good y Kaminski, 2002). Su principal finalidad es evaluar el progreso de aprendizaje de la población escolar canaria identificada con "riesgo" de presentar dificultades específicas de aprendizaje en lectura y que participa en un Modelo de Respuesta a la Intervención.

En la actualidad, la Ley Orgánica 2/2006, de 3 de mayo, de Educación (LOE) regula la educación de los estudiantes en todo el territorio español, estableciendo la atención a la diversidad como principio básico del sistema educativo para atender a todas las etapas educativas y a todos los alumnos. De este modo, lo que se trata es de responder adecuadamente a las demandas educativas que la diversidad de alumnos requiere, sin ningún tipo de exclusión. Bajo este marco legal, la Comunidad Autónoma de Canarias, a través de la Consejería de Educación, Universidades, Cultura y Deportes en el Decreto 104/2010, establece el marco normativo que regula en el ámbito de la Comunidad Autónoma de Canarias la atención a la diversidad, especialmente, la atención educativa al alumnado que presente necesidades específicas de apoyo educativo. Dentro de este grupo, se encuentra el alumnado con Dificultades Específicas de Aprendizaje donde se sitúan, más concretamente, las Dificultades Específicas de Aprendizaje de Lectura o Dislexia. Según la norma Canaria, un alumno o alumna se identifica con las "Dificultades específicas de aprendizaje en lectura o dislexia" cuando muestra los siguientes indicadores: una competencia curricular en lectura con un retraso de dos cursos, al menos, respecto al nivel que le correspondería por su edad cronológica, además de problemas asociados a la adquisición de la ortografía y al deletreo, así como un rendimiento normal mediante pruebas estandarizadas de escritura y cálculo, salvo que el bajo rendimiento se deba a una disgrafía o discalculia; este alumnado acredita un cociente intelectual superior a 80 en tests de inteligencia general; un bajo rendimiento en tests estandarizados de lectura respecto al curso que le correspondería por edad, con un percentil inferior a 25 en lectura de pseudopalabras, o un percentil mayor o igual a 75 en tiempos de lectura de palabras o pseudopalabras. Después de constatados los criterios anteriores, el escolar podría presentar, en ocasiones, un percentil inferior a 50 en pruebas estandarizadas de comprensión lectora.
El Programa para la Atención Educativa del Alumnado con Dificultades Específicas de Aprendizaje menciona la puesta en práctica de instrumentos, estrategias y recursos para la detección temprana y la adecuación de la respuesta educativa a las necesidades de dicho alumnado. Se hace necesario que se diseñen, experimenten y evalúen instrumentos y procedimientos de detección temprana e identificación, así como programas de intervención educativos aplicables en los centros escolares por los profesionales presentes en los mismos. Se pretende un mejor entrenamiento del profesorado con modelos de intervención eficaces desarrollados en nuestro contexto. Según Jimenez (2010), en los últimos años estamos asistiendo a un cambio sustantivo de orientación en el campo científico de las dificultades de aprendizaje con implicaciones directas tanto en la identificación como en el tratamiento. La implementación del modelo tradicional basado en el uso de la discrepancia CIrendimiento está dejando paso a un modelo alternativo basado en la respuesta a la intervención, gracias a la implantación de la Ley IDEA, de 2004 (Ley de Educación para Personas con Discapacidades) en EEUU, que está teniendo una influencia decisiva en el campo de las dificultades de aprendizaje.

\section{Modelo de Respuesta a la Intervención (RtI)}

El modelo de respuesta a la intervención surge como alternativa al criterio de discrepancia en la "Ley de Educación para personas con Dificultades" (Individuals with Disabilities Education Improvement Act, IDEA, 2004). Hasta ese momento se instaba a los profesionales a utilizar el criterio de discrepancia CI-rendimiento para el diagnóstico de dificultades específicas de aprendizaje (DEA). Sin embargo, en esta ley se da la posibilidad de utilizar un método alternativo, el modelo de respuesta a la intervención (RtI). El modelo de respuesta a la intervención, a diferencia del criterio de discrepancia, está centrado en los alumnos con riesgo de padecer DEA. Es decir, con este modelo se trata de prevenir y anticipar las dificultades realizando una identificación temprana y una evaluación progresiva de la respuesta del niño. Según este modelo todos los niños deben ser evaluados tempranamente, tratando de distinguir aquellos que puedan tener mayores dificultades que su grupo de referencia (niños con riesgo). A continuación debe llevarse un control de la respuesta del alumno a la intervención para la mejora de sus habilidades, la cual debe estar basada en la evidencia científica (Vaughn y Fuchs, 2003). Aquellos alumnos que no respondan a la intervención científica serán remitidos para ser evaluados por los equipos de orientación.

El modelo RtI lleva implícito la intervención como forma de evaluación. Es necesario llamar la atención sobre el hecho de que las intervenciones en los niños con riegos son planificadas y basadas en la investigación científica en el área de las dificultades de aprendizaje. De esta manera podemos asegurar que si los niños muestran resistencia a esta intervención sus dificultades no se derivan de una instrucción inadecuada. Son muchos los modelos propuestos, sin embargo todos coinciden 
en su naturaleza multinivel. Un primer nivel consiste en una instrucción generalizada al grupo de clase, y que va aumentando en nivel, o lo que es lo mismo, en intensidad (entendida como tiempo invertido en la instrucción y tipo de agrupamiento) según la respuesta del niño a dicha intervención.

Uno de los aspectos cruciales a tener en cuenta en este modelo es la detección de los niños con riesgo de padecer DEA en edades tempranas (5-7 años). La detección precoz está justificada, a partir de los hallazgos obtenidos en diferentes estudios llevados a cabo, donde se demuestra que los niños que se detectan tempranamente y reciben una intervención adecuada tienen un mejor pronóstico que los que son detectados en edades más avanzadas (Wanzek y Vaughn, 2007). Esta fase inicial de "screening" en la Comunidad Autónoma de Canarias es realizada a través del The Hong Kong Specific Learning Difficulties Behavior Checklist (Ho, Chan, Tsang y Lee, 2002; ver traducción y adaptación al español en Jiménez y Artiles, 2001) que han de cumplimentar los profesores. En la figura 1, presentamos un ejemplo de modelo de respuesta a la intervención con tres niveles.

Figura 1. Modelo de tres niveles de aumento en intensidad académica. Los porcentajes hacen referencias al número de niños estimados que están en el Nivel 1 y que requieren Nivel 2 y Nivel 3 de servicio. (Tomado y adaptado de National Association of State Directors of Special Education, 2006).

\section{SISTEMA ACADÉMICO}

Nivel 3. Intervención individualizada e intensiva:

- Estudiantes de forma individualizada

- Evaluación base

- Gran intensidad

Nivel 2. Intervención centrada pequeño grupo: - Algunos estudiantes de riesgo

- Gran eficiencia

- Respuesta rápida

Nivel 1. Intervención instruccional: - Todos los estudiantes

- Preventiva y proactiva

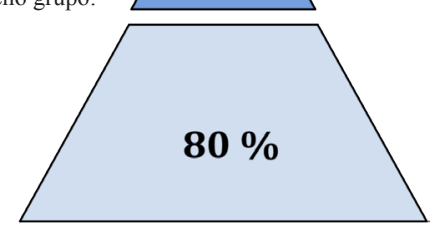

ESTUDIANTES

Principales componentes o habilidades de lectura a instruir En 1997, el Congreso de EE.UU solicitó al NICHD (Instituto Nacional de Salud Infantil y Desarrollo Humano) junto con el Departamento de Educación, la formación del National Reading Panel (NRP) para revisar investigaciones sobre cuáles serían los principales componentes que están a la base del aprendizaje de la lectura y determinar qué métodos de enseñanza de la lectura basados en la evidencia empírica serían más eficaces. Una de las principales conclusiones de este informe fue que el mejor enfoque para la enseñanza de lectura es aquel que incorpora la instrucción explícita de la conciencia fonológica, el principio alfabético, actividades para mejorar la fluidez, el vocabulario y la comprensión (NRP, 2000). Así, el análisis del NRP definió las Cinco Grandes Ideas (Five Big Ideas): conciencia fonémica, conocimiento alfabético, fluidez, vocabulario y comprensión.

\section{Conciencia fonémica}

Los niños deben aprender a descubrir que las palabras habladas están compuestas de partes más pequeñas llamadas fonemas. La instrucción de la conciencia fonémica proporciona a los niños un fundamento básico que les ayuda a aprender a leer y escribir. El NRP encontró que los niños que aprendieron a leer a través de la instrucción específica en conciencia fonémica mejoraban sus habilidades de lectura más que aquellos que aprendieron en programas donde no se prestaba atención alguna a esta habilidad metalingüística. Lyon (1995) encontró que el mejor predictor que explicaba la dificultad para aprender a leer en kindergarten y en primer grado era la incapacidad de segmentar palabras y sílabas en unidades constituyentes de sonidos. Según Smith, Simmons y Kame'ennui (1998) la capacidad para escuchar y manipular los fonemas juega un papel causal en la adquisición de las habilidades iniciales de la lectura. Por su parte, Shaywitz (2003) encontró que los efectos del entrenamiento en conciencia fonémica y la lectura de palabras se refuerzan mutuamente. Es decir, que la conciencia fonémica es necesaria para la lectura y la lectura, a su vez, mejora aún más la conciencia fonémica. En castellano, la conciencia fonémica es tan importante como en inglés ya que en la mayor parte de los casos a cada letra del alfabeto le corresponde un sonido. Resultados de varias investigaciones han indicado que los alumnos que carecen de conciencia fonémica tienden a presentar dificultades posteriores en la lectura (Bravo-Valdivieso, 1995; Carrillo, 1994; Jiménez 1997; Jiménez et al., 2005).

\section{Conocimiento alfabético}

La instrucción orientada a desarrollar habilidades fonológicas incide en la relación entre fonemas y las letras impresas, y considera fundamental el uso de este conocimiento para aprender a leer y deletrear. El NRP encontró que los estudiantes muestran marcados beneficios en la instrucción explícita del conocimiento alfabético desde kindergarten hasta sexto curso. Según Juel (1991) el conocimiento de las reglas de conversión grafema-fonema (CGF) es un prerrequisito para la identificación eficaz de la palabra. La principal diferencia entre los buenos lectores y los que no lo son, es la capacidad de utilizar la correspondencia entre letras y sonidos para identificar las palabras. En este sentido, ya Ehri (1991) señalaba que durante la fase alfabética (es decir la fase en donde el niño reconoce las letras como símbolos para los sonidos), la lectura debe incluir mucha práctica en la descodificación fonológica de las mismas palabras para que el alumno alcance a familiarizarse con sus patrones ortográficos. En los estudios llevados a cabo por Stanovich (1986), se llegaba a la conclusión de que los estudiantes que adquieren y aplican de manera temprana el principio alfabético en la lectura, obtienen mayores beneficios a largo plazo. Más tarde, Liberman y Liberman (1990) señalaron que enseñar a los estudiantes a descodificar fonológicamente las palabras es una tarea difícil, pero que es una meta alcanzable 
y con efectos de larga duración. Y, finalmente, los estudios de Haskell, Foorman y Swank (1992) demostraron que la combinación de una instrucción en conciencia fonémica y el uso de las reglas de CGF, parece ser más favorable para el éxito en la lectura temprana. Dado que el español tiene un sistema ortográfico con gran transparencia (i.e., que cada letra representa un sonido), los alumnos se ven beneficiados por el hecho de que el nivel de ambigüedad en la relación grafema fonema es muy baja, de manera que con aprenderse un número limitado de correspondencias y ciertas reglas grafo-fonémicas los niños ya pueden leer cualquier palabra.

\section{Fluidez}

La fluidez significa ser capaz de leer con rapidez y precisión textos, utilizando la entonación apropiada dentro del contexto de la lectura. La instrucción en la fluidez incluye la lectura oral guiada, en la que los alumnos leen en voz alta a alguien que corrige sus errores y les proporciona retroalimentación. El NRP encontró que la lectura fluida mejora las habilidades de los estudiantes para reconocer nuevas palabras, leer con mayor rapidez, precisión y expresión, y comprender mejor lo que leen. Según Hasbrouck (1998) los buenos lectores se basan principalmente en las letras de la palabra en lugar del contexto o ilustraciones para identificar palabras conocidas y desconocidas. Utilizan las reglas de CGF para identificar las palabras y leen las palabras un número suficiente de veces para que el reconocimiento de las palabras se convierta en algo automático. Como habían sugerido Kuhn y Stahl (2000): “los lectores competentes son tan automáticos con cada habilidad (conciencia fonológica, descodificación, vocabulario) que centran toda su atención en construir el significado de la impresión". Hay suficiente evidencia científica que indica que la fluidez en la lectura oral es una medida efectiva de la capacidad lectora de los niños en todas sus dimensiones, incluyendo la comprensión (Baker et al., 2008; Deno, Mirkin y Chiang, 1982; Dominguez de Ramírez y Shapiro, 2007; Fuchs, Fuchs y Maxwell, 1988; Hamilton y Shinn, 2003). Aunque a veces la fluidez en la lectura oral se equipare a la lectura rápida (i.e., se incentiva al alumno a leer las palabras rápidamente), la fluidez en la lectura oral también abarca una serie de habilidades lectoras que incluyen (a) la identificación automática de la relación entre letras y sonidos, (b) la habilidad de formar una unidad de estos sonidos en combinaciones reconocibles, (c) el acceso automático a las representaciones léxicas, (d) el reconocimiento de conexiones significativas dentro de oraciones y entre oraciones, (e) la habilidad de asociar el significado del texto con información dada anteriormente, y (f) la deducción del contenido que pueda faltar en el texto (Fuchs, Fuchs, Hosp y Jenkins, 2001).

\section{Comprensión y Vocabulario}

La instrucción en vocabulario se ha de centrar en facilitar a los estudiantes no solo el reconocimiento de las palabras sino también en entenderlas a través de su uso. El NRP encontró que la enseñanza de vocabulario y el contacto repetido con el uso de palabras es sumamente importante para el éxito escolar, en general, y para la comprensión de la lectura en particular (ver también, Anderson y Nagy, 1991). En la misma línea, The National Research Council (1998) concluyó que el desarrollo del vocabulario es un objetivo fundamental para los estudiantes en los primeros grados. Baker, Simmons y Kame'enui (1997) demostraron que los niños que se incorporaban con un conocimiento limitado del vocabulario a la escuela experimentaban mayores dificultades en comparación con sus compañeros que tenían un conocimiento rico del vocabulario. "Un estudiante promedio en los grados 3-12 es probable que aprenda unas 3.000 palabras de vocabulario nuevas cada año, suponiendo que él o ella lea entre 500.000 y un millón de palabras de texto en un año escolar". Entre los grados 1 y 3, se estima que el «aumento de vocabulario está en torno a 3.000 palabras por año en los estudiantes de clase media». El tamaño del vocabulario infantil se duplica entre los grados 3 y 7. El desarrollo del vocabulario masivo parece ocurrir sin mucha ayuda de los maestros (Nagy y Anderson, 1984). Posteriormente, Anderson y Nagy (1992) demostraron que los niños que alcanzan a leer diez minutos al día fuera de la experiencia escolar presentan mayores tasas de crecimiento de vocabulario entre los grados segundo y quinto que los niños que practican la lectura poco o nada. Y, en relación a la comprensión, el NRP (2000) aportó que los lectores que comprenden bien no son únicamente buenos descodificadores. También encontró que el tiempo dedicado a la lectura está altamente correlacionado con la comprensión.

\section{Evaluación del progreso de aprendizaje}

La implementación del modelo RtI lleva implícito evaluar de forma temprana la respuesta de un alumno o alumna que presenta suficientes indicadores de obstáculos para sus aprendizaje futuro, la aplicación de un programa de intervención personalizado, basado en la investigación científica, que coincida con sus necesidades individuales, con el fin de ir tomando decisiones educativas en función de la evolución de su aprendizaje. Se trata de evaluar adecuadamente la respuesta del alumno a dicha instrucción e intervención. Un factor clave en este modelo es el control regular del rendimiento del alumno para comprobar el avance o resistencia a la intervención (Fuchs y Fuchs, 2006). A raíz de la implementación del Modelo RtI en EE.UU monitorizar el progreso de aprendizaje de los alumnos es una parte central de este modelo a la hora de tomar decisiones para la identificación y la repuesta educativa en el ámbito de las necesidades educativas específicas (Fuchs y Fuchs, 2006; Speece, Case y Molloy, 2003). En este sentido un sistema de evaluación que se viene utilizando en el ámbito norteamericano ha sido el Dynamic Indicators of Basic Early Literacy Skills (DIBELS) cuya versión en español es el IDEL (Indicadores Dinámicos del Éxito en la Lectura). 
La investigación inicial sobre DIBELS se llevó a cabo en la Universidad de Oregon a finales de 1980. Desde entonces, una serie de estudios sobre DIBELS ha documentado la fiabilidad y la validez de las medidas, así como su sensibilidad al cambio del rendimiento del estudiante. Asimismo, también se cuenta con documentación similar sobre las propiedades psicométricas de la versión española del IDEL (ver por ejemplo, Baker, 2009; Baker, Cummings, Good y Smolkowski, 2007; Baker, Park y Baker, 2010a, 2010b; Watson, 2004). Se trata de un conjunto de procedimientos y medidas para evaluar la adquisición de las habilidades de alfabetización temprana desde el periodo de Educación Infantil (5 años) hasta segundo curso. Están diseñados para ser breves (un minuto) y utilizando medidas de fluidez para controlar regularmente el desarrollo de la alfabetización y las habilidades de lectura temprana (Good y Kaminski, 2002).

El sistema DIBELS fue desarrollado para medir las habilidades relacionadas con la lectura, que son reconocidas y validadas empíricamente. Cada medida ha sido investigada con exhaustividad y se ha demostrado que incorpora indicadores fiables y válidos para el desarrollo de la alfabetización temprana y la predicción del retraso en la lectura, ayudando también a la identificación temprana de los estudiantes que no están avanzando como se esperaba. Cuando se utiliza según lo recomendado, los resultados pueden ser utilizados para evaluar el desarrollo individual del estudiante, así como para proporcionar información del nivel logrado en relación con los objetivos de instrucción validados.

En general, medir el progreso de aprendizaje de los alumnos en cada uno de los componentes que hemos citado previamente ha de ser un objetivo prioritario dentro del proceso educativo. En este sentido, monitorizar el progreso de aprendizaje y utilizar medidas basadas en el currículo se ha propuesto como una forma de supervisar el progreso de aprendizaje de acuerdo a normas académicas standard definidas por los propios organismos o administraciones educativas en EEUU (Shinn, 1989; Wallace, Espin, McMaster, Deno y Foegen, 2007). Como sugieren estos mismos autores, el área de lectura ha recibido mucha más atención que la escritura o las matemáticas (ver para una revisión Marston, 1989; Wallace et al, 2007) y la aplicación de este sistema de evaluación a estudiantes con dificultades se ha estudiado mucho más que su aplicación a otras categorías diagnósticas. Las medidas IDEL han demostrado ser fiables y válidas, y permiten además medir el progreso de aprendizaje a partir de datos normativos. Justamente, el principal propósito que persigue este sistema de evaluación es proporcionar a los educadores normas para medir el progreso de todos los estudiantes que reciben instrucción en lectura en español, pero de manera individual. Los benchmark o puntos de referencia representan los niveles mínimos de rendimiento que todos los estudiantes deben poseer para considerar que se encuentran en camino de convertirse en buenos lectores. Los objetivos del sistema y los puntos de corte se basan en la investigación.
Éstos indican la probabilidad de lograr los objetivos en la alfabetización temprana. Los puntos de referencia para cada medida y los momentos de medida (Inicio, Medio, Fin) se establecieron mediante un punto de corte mínimo en el que las probabilidades de lograr el objetivo de referencia siguiente se encuentra a favor del estudiante. Para que una puntuación pueda ser considerada un objetivo de referencia, al menos del $80 \%$ al $85 \%$ de los estudiantes en la muestra tienen que lograr esa puntuación en ese momento para luego lograr la próxima meta. Así, para un niño con una puntuación igual o superior al objetivo de referencia, la probabilidad para lograr la siguiente meta será alta y la probabilidad de necesidad de apoyo adicional para alcanzar la próxima meta será baja. Además de estos objetivos, el sistema también incluye los puntos de corte, donde se indican las probabilidades de alcanzar los objetivos de la alfabetización posterior. Estos puntos de corte representan las puntuaciones en las cuales el $20 \%$ o menos de los estudiantes suelen alcanzar los objetivos siguientes. Los estudiantes con calificaciones iguales o por debajo de estos puntos de corte tienen muy pocas posibilidades de alcanzar los objetivos de la alfabetización posterior, a menos que se proporcione un apoyo adicional en la instrucción. Una característica de las normas de decisión sobre los puntos de referencia DIBELS, o en su versión en español IDEL, es la inclusión de una zona en la que no es posible una predicción clara. Las puntuaciones que caen entre el objetivo de referencia y el punto de corte representan patrones de rendimiento en donde aproximadamente un 50\% de los estudiantes lograron los objetivos de la alfabetización posterior. Los estudiantes con calificaciones en esta categoría requieren de una planificación estratégica por parte de los educadores para determinar las estrategias apropiadas que apoyen a los estudiantes para cumplir con los siguientes objetivos.

En definitiva, estas medidas que incluye el sistema de evaluación DIBELS e IDEL fueron diseñadas específicamente para evaluar las Grandes Ideas (Big Ideas) de la alfabetización temprana propuestas por el NRP (2004): la conciencia fonológica, el principio alfabético, la fluidez, vocabulario y comprensión. Combinadas, las medidas forman un sistema de evaluación del desarrollo de la alfabetización temprana que permite a los educadores determinar el progreso de aprendizaje en lectura del estudiante de manera fácil y fiable (Baker, Cummings, Good y Smolkowski, 2007; Good y Kaminski, 2002). Se recomienda evaluar a los estudiantes al principio, medio y final del año académico para permitir la retroalimentación de resultados sobre la instrucción y hasta el momento no se disponen de datos normativos sobre el progreso de aprendizaje en población escolar canaria. Es evidente que la validez ecológica de los resultados de la evaluación, cuando se trata de comparar el rendimiento individual con grupos de referencia que pertenecen a otros contextos idiomáticos y culturales, donde incluso los sistemas educativos pueden no ser equivalentes, estaría siempre comprometida. Por ello, la 
principal finalidad que nos hemos trazado en la Comunidad Autónoma de Canarias ha sido la de medir el progreso de aprendizaje en la población escolar canaria que no se encuentra en situación de "riesgo" de presentar DEAL, para cada uno de los indicadores que incluye IDEL como sistema de evaluación. Con la obtención de estos datos normativos será posible interpretar de forma más adecuada el progreso de aprendizaje que experimentan los alumnos que han sido identificados en situación de "riesgo" y que están participando en la actualidad en un programa institucional basado en la implementación de un modelo de respuesta a la intervención que promueve la Consejería de Educación, Universidades y Sostenibilidad del Gobierno de Canarias y que se extiende a muchos centros escolares que se encuentran distribuidos en la Comunidad Autónoma de Canarias (ver Jiménez, 2010; Jiménez et al., 2010).

Este instrumento cuenta con un cuadernillo diferente para cada alumno según el curso académico (Educación Infantil de 5 años, $1^{\circ}$ y $2^{\circ}$ Educación Primaria) en el que se registra el resultado de cada indicador en cada momento de medida. Existe una guía para la administración y calificación de IDEL (Cummings, Baker y Good, 2006). El examinador tiene además una carpeta con los estímulos que se le van a presentar al niño y un cronómetro. Cada prueba evalúa una destreza y su duración es de un minuto: 1) Fluidez en nombramiento de letras: evalúa la habilidad de un niño para identificar y producir el nombre de una letra dada. Se le facilita al alumno una plantilla con 100 letras, mezclando mayúsculas, minúsculas y distintos tipos de caligrafía, y el cometido del niño es leer el mayor número de letras posibles y de manera correcta. Se obtiene la puntuación directa de las letras leídas correctamente; 2) Fluidez de Segmentación de Fonemas (FSF): evalúa la habilidad de un niño para la producción de sonidos individuales dentro de una palabra dada. Se presentan 24 palabras oralmente y el alumno debe identificar todos los sonidos de cada palabra y de manera ordenada. Se contabilizan los sonidos correctos expresados por el niño (se tienen en cuenta las distintas unidades de segmentación lingüística: fonemas, lexemas, morfemas, sílabas, etc.); 3) Fluidez en Palabras sin Sentido (FPS): evalúa los conocimientos de un niño en las correspondencias letra-sonido y su capacidad para combinar letras para formar palabras «sin sentido»o pseudopalabras. La labor del niño es leer el mayor número de pseudopalabras posible y de manera correcta en el tiempo indicado. Se obtiene la puntuación directa de las 60 pseudopalabras leídas correctamente. Ya que las palabras de mayor frecuencia en castellano tienen sílabas de consonante vocal, todas las pseudopalabras en IDEL FPS son de una sílaba (CV) o de dos sílabas (CVCV); 4) Fluidez en la Lectura Oral (FLO): evalúa la habilidad de un niño en la lectura de un texto con palabras de uso frecuente en base a su edad. El texto cuenta con aproximadamente 200 palabras y el nivel de dificultad es directamente proporcional al nivel escolar. Todos los textos se escribieron tomando en cuenta la longitud de las oraciones, el número de palabras frecuentes y el número de letras y sílabas en palabras. Se contabilizan el número de palabras correctas e incorrectas leídas por el alumno; y 5) Fluidez en el Uso de la Palabra (FUP): evalúa la capacidad del niño para utilizar con precisión una palabra en el contexto de una frase. Se le pide al niño que haga una frase con cada palabra que le digamos. Esta prueba consta de 18 palabras y se contabiliza el número total de palabras que utiliza en las frases de manera correcta. Todas las palabras para cada grado se tomaron al azar de varios libros de texto escolares en castellano.

Ya que los datos normativos de las medidas que incluye el sistema de evaluación IDEL se obtuvieron con población hispana que vive en Estados Unidos (Baker, Cummings, Good y Smolkowski , 2007), una de las tareas a realizar ha sido obtener datos normativos en población canaria en tres momentos diferentes a lo largo del curso escolar 2010-2011.

\section{Participantes \\ Método}

La muestra utilizada para llevar a cabo esta tarea estaba formada por 758 niños, (370 niños y 388 niñas), con un rango de edad comprendido entre los 5 y los 7 años, pertenecientes a los cursos de 5 años de Educación Infantil, $1^{\circ}$ y $2^{\circ}$ de Educación Primaria, procedentes de seis escuelas públicas y concertadas, situadas en los municipios de Santa Cruz de Tenerife y La Laguna (Área Metropolitana). Se controló que hubiese una distribución homogénea de la muestra según nivel escolar y sexo, y como criterios de exclusión de la muestra, los alumnos participantes no presentaban discapacidades intelectuales, sensoriales, físicas, o motoras.

\section{Procedimiento}

Las medidas se administraron con un espacio de aproximadamente tres meses entre cada una de ellas. La primera medida fue tomada en el mes de diciembre, la segunda en marzo y la tercera en junio. Se trasladó a los centros un equipo de 12 examinadores y la distribución se realizó conforme a dos examinadores por colegio. Este equipo de examinadores recibió una formación tanto teórica como práctica sobre el sistema de evaluación IDEL durante una semana. Posteriormente y coincidiendo con la finalización de cada medida, se concretaba una reunión para la revisión del trabajo y aclaración de dudas sobre la corrección de las pruebas por parte de los examinadores. El tiempo de evaluación para cada uno de los indicadores medidos a través del IDEL era siempre de un minuto y para ello los examinadores disponían cada uno de ellos de un cronómetro.

\section{Análisis de los datos}

Se calcularon las puntuaciones para cada una de las medidas del sistema de evaluación IDEL (i.e., fluidez en nombrar letras, fluidez en la segmentación de fonemas, fluidez de palabras sin 
Figura 2. Indicadores del sistema de evaluación IDEL para cada momento de medida y nivel escolar.

\section{IDEL $^{\circledast}$}

Fluidez en la Lectura Oral

\begin{tabular}{|c|c|c|c|c|c|c|}
\hline \multicolumn{4}{|c|}{ Fluidez en la Lectura Oral } \\
\hline
\end{tabular}

sentido, fluidez en lectura oral y fluidez en el uso de palabras). La Figura 2 recoge qué medidas se aplican en función del curso (Educación Infantil 5 años, $1^{\circ}$ y $2^{\circ}$ Educación Primaria) y del momento de medida (Inicio, Medio y Fin).

Para el curso de Educación Infantil 5 años, los indicadores que se evalúan en los tres momentos de medida son: fluidez en nombramiento de letras, fluidez en segmentación de fonemas, y fluidez en el uso de las palabras. En cambio, la fluidez en palabras sin sentido es evaluada solamente en dos momentos de medida: medio y fin. Con respecto al $1^{\circ}$ curso de Educación Primaria los indicadores que se evalúan en los tres momentos de medida son: fluidez en la segmentación de fonemas, fluidez en palabras sin sentido, y fluidez en el uso de palabras. Mientras que la medida de fluidez en nombramiento de letras se realiza sólo al inicio, las medidas de fluidez en lectura y relato oral se recogen en dos momentos de medida: medio y fin. Y, finalmente, en $2^{\circ}$ curso de Educación Primaria, los indicadores que se evalúan en los tres momentos de medida son: fluidez en lectura y relato oral y fluidez en el uso de palabras. En cambio, fluidez en palabras sin sentido se evalúa sólo en la fase de inicio.

\section{Resultados}

Atendiendo a los datos normativos que se obtienen a través del sistema de evaluación IDEL, se establece que aquellos alumnos cuya puntuación coincida o se sitúe por debajo del Pc20 deberían ser considerados en situación de "riesgo" en relación al nivel de desarrollo que están mostrando en la habilidad medida. Si la puntuación se sitúa entre los Pc20 y Pc40, debería ser considerado en situación de "riesgo medio" en la habilidad medida y, finalmente, si la puntuación se ubica entre los Pc40 y Pc60, debería ser considerado en situación de "bajo riesgo" en la habilidad medida.

\section{Discusión}

En síntesis, el objetivo principal del trabajo que aquí presentamos ha sido describir el sistema de evaluación del progreso de aprendizaje de la lectura dentro de un modelo RtI que se viene implementando en la Comunidad Autónoma de Canarias (Jiménez et al., 2010). Tanto la implementación del modelo RtI como el sistema de evaluación IDEL está siendo posible gracias al convenio de cooperación científica entre el grupo de investigación de la ULL "Dificultades de aprendizaje, psicolingüística y nuevas tecnologías", el Center on Teaching and Learning (CTL) de la Universidad de Oregon y la Consejería de Educación, Universidades y Sostenibilidad del Gobierno de Canarias. Dentro de este contexto se han obtenido datos normativos para población escolar canaria sobre el sistema de evaluación Indicadores Dinámicos para el Éxito en la Lectura (IDEL, Baker et al. 2006) versión española del Dynamic Indicators of Basic Early Literacy Skills (DIBELS, Good y Kaminski, 2002). Estos datos normativos se han calculado para cada una de las medidas que recoge el sistema IDEL de evaluación del progreso de aprendizaje en la lectura en función del curso y del momento de evaluación. Hasta ahora los análisis realizados sobre los distintos indicadores que recoge el sistema de evaluación nos permiten constatar que el instrumento es sensible a los cambios que tienen lugar durante el proceso de aprendizaje de la lectura. En suma, con la obtención de datos normativos para cada una de las medidas de progreso de aprendizaje de la lectura en función del curso y del momento de evaluación los profesores, en general, y los profesores especialistas de apoyo y refuerzo a las NEAE, en particular, podrán evaluar a partir de ahora el progreso de aprendizaje de los niños canarios que son identificados con "riesgo" de presentar DEAL y que participan en un Modelo de Respuesta a la Intervención que se implementa en la Comunidad Autónoma de Canarias. 


\section{Referencias}

1. Anderson, R.C. y Nagy, W.E. (1991). Word meanings. E.P. y Pearson, P.D. (Eds.), Handbook of Reading Research, Vol. II (pp.789-814). New York: Longman.

2. Anderson, R. C. y Nagy, W. E. (1992). The vocabulary conundrum. The American Educator, 16, 14-18. http://dx.doi. org/10.2307/3193321

3. Baker, D.L. (2009). Predictive and concurrent criterionrelated validity of the indicadores dinámicos del éxito en la lectura for students learning to read in Spanish in Mexico. (Technical Report) Eugene, OR: Dynamic Measurement Group.

4. Baker, D. L., Cummings, K. D., Good, R. H. y Smolkowski, K. (2007). Indicadores dinámicos del éxito en la lectura (IDEL $\AA)$ : Summary of decision rules for intensive, strategic, and benchmark instructional recommendations in kindergarten through third grade (Technical Report No.1) Eugene, OR: Dynamic Measurement Group.

5. Baker, D. L., Park, Y. y Baker, S.K. (2010a). The reading performance of English learners in grades 1-3: the role of initial status and growth on reading fluency in Spanish and English. Reading and Writing, 1-31. (Online First).

6. Baker, D. L., Park, Y. y Baker, S. K. (2010b). Effect of initial status and growth in pseudoword reading on Spanish reading comprehension at the end of first grade. Psicothe$m a, 22,955-962$.

7. Baker, S.K., Simmons, D. y Kame'enui, E.J. (1997). Vocabulary acquisition: Research bases. En D.C. Simmons y E.J. Kame'enui (Eds.), What reading research tells us about children with diverse learning needs: Bases and basics. Mahwah, NJ: Erlbaum.

8. Baker, S. K., Smolkowski, K., Katz, R., Fien, H., Seeley, J. R., Kame'enui, E. J. y Beck, C. T. (2008). Reading fluency as a predictor of reading proficiency in low-performing, high-poverty schools. School Psychology Review, 37, 1837.

9. Bravo-Valdivieso, L. (1995). A four year follow-up study of low socioeconomic status, Latin American children with reading difficulties. International Journal of Disability, Development and Education, 42, 189-202. http://dx.doi. org/10.1080/0156655950420302

10. Carrillo, M. (1994). Development of phonological awareness and reading acquisition. A study in Spanish language. Reading and Writing: An Interdisciplinary Journal, 6, 279298. http://dx.doi.org/10.1007/BF01027086

11. Cummings, K. D., Baker, D. L. y Good, R. H. (2006). Guía para la administración y calificación de IDEL. En R. H. Good, D. L. Baker, N. Knutson, y J. M. Watson (Eds.), Indicadores dinámicos del éxito en la lectura (7a ed.). Eugene, OR: Dynamic Measurement Group.

12. Deno, S.L., Mirking, P.K. y Chiang, B. (1982). Identifying valid measures of reading. Exceptional Children, 49, 36-45.

13. Domínguez de Ramírez, R. y Shapiro, E. S. Cross-langua- ge relationship between Spanish and English oral reading fluency among Spanish-speaking English language learners in bilingual education classrooms. Psychology in the Schools, 44, 795-806. http://dx.doi.org/10.1002/pits.20266

14. Ehri, L. (1991). Development of the ability to read words. En R. Barr, M. L. Kamil, P. Mosenthal \& P. D. Pearson (Eds.), Handbook of Reading Research (pp. 383-417). Nueva York: Longman.

15. Fuchs, L. S. y Fuchs, D. (2006). Identifying learning disabilities with RTI. Perspectives, 32, 39-43.

16. Fuchs, L. S., Fuchs, D., Hosp, M. K. y Jenkins, J. R. (2001). Oral reading fluency as an indicator of reading competence: A theoretical, empirical, and historical analysis. Scientific Studies of Reading, 5, 241-258. http://dx.doi.org/10.1207/ $\underline{\mathrm{S} 1532799 X S S R 0503 \quad 3}$

17. Fuchs, L.S., Fuchs, D. y Maxwell, L. (1988). The validity of informal reading comprehension measures. Remedial and Special Education, 9, 20-38. http://dx.doi. org/10.1177/074193258800900206

18. Good, R. H., Knutson, N. y Watson, J. M. (Eds.) (2006). Indicadores dinámicos del éxito en la Lectura (7a ed.). Eugene, OR: Dynamic Measurement Group, Inc. Acceso: http:// dibels.uoregon.edu/.

19. Good, R. H. y Kaminski, R. A. (Eds.) (2002). Dynamic indicators of basic early literacy skills (6th ed.). Eugene, OR: Institute for the Development of Educational Achievement. Available: http://dibels.uoregon.edu/

20. Hamilton, C. y Shinn, M.R. (2003). Characteristics of word callers: An investigation of the accuracy of teachers' judgments of reading comprehension and oral reading skills. School Psychology Review, 32, 228-240.

21. Hasbrouck, J. (1998). Reading fluency: Principles for instruction and progress monitoring. Professional Development Guide. Austin, TX: Texas Center for Reading and Language Arts, University of Texas at Austin.

22. Haskell, D. W., Foorman, B. R. y Swank, P. R. (1992). Effects of three orthographic/phonological units on firstgrade reading. Remedial and Special Education, 13, 40-49. http://dx.doi.org/10.1177/074193259201300207

23. Ho, C.S.H., Chan, D.W.O., Tsang, S.M. y Lee, S.H. (2002). The Hong Kong test of specific learning difficulties in reading and writing (HKT-SpLD). Hong Kong: Hong Kong Specific Learning Difficulties Research Team.

24. Individuals with Disabilities Education Improvement Act, 20 U.S.C. 1400 et seq. (2004).

25. Jiménez, J.E. (1997). A reading-level match study of phonemic processes underlying reading disabilities in a transparent orthography. Reading and Writing: An Interdisciplinary Journal, 9, 23-40.

26. Jiménez, J.E. (2010). Response to Intervention (RtI) Model: A promising alternative for identifying students with learning disabilities? Psicothema, 22, 932-934.

27. Jiménez, J.E. y Artiles, C. (2011). Adaptación al español 
del Test The Hong Kong Specific Learning Difficulties Behavior Checklist. Guía para la detección temprana de discapacidades, trastornos y dificultades de aprendizaje. Consejería de Educación, Universidades, Cultura y Deportes del Gobierno de Canarias.

28. Jiménez, J.E., García, E., Ortiz, M.R., Hernández-Valle, I., Guzmán, R., Rodrigo, M., Estévez, A., Díaz, A. y Hernández, S. (2005). Is the deficit in phonological awareness better explained in terms of task differences or effects of syllable structure? Applied Psycholinguistics, 26, 267-283.

29. Jiménez, J.E., Rodríguez, C., Crespo, P., González, D., Artiles, C. y Afonso, M. (2010). Implementation of Response to Intervention (RtI) Model in Spain: An example of a collaboration between Canarian universities and the department of education of the Canary Islands. Psicothema, 22, 935-942.

30. Juel, C. (1991). Beginning reading. En R. Barr, M. L. Kamil, P. B. Mosenthal, y P. D. Pearson (Eds.), Handbook of reading research (pp. 759-788). New York: Longman.

31. Kuhn, M. R. y Stahl S.A. (2000). Fluency: A review of developmental and remedial practices. Ann Arbor, MI: Center for the Improvement of Early Reading Achievement.

32. Liberman, I. Y. y Liberman, A. M. (1990). Whole language vs. code emphasis: Underlying assumptions and their implications for reading instruction. Annals of Dyslexia, 40, 51-76. http://dx.doi.org/10.1007/BF02648140

33. Lyon, G. R. (1995). Toward a definition of dyslexia. Annals of Dyslexia, 45, 3-27. http://dx.doi.org/10.1007/ BF02648210

34. Nagy, W. y Anderson, R. C. (1984). How many words are there in printed school English? Reading Research Quarterly, 19, 304-330. http://dx.doi.org/10.2307/747823

35. Marston, D. (1989). A curriculum-based measurement approach to assessing academic performance: What it is and why do it. En M.Shinn (Ed.), Curriculum-based measurement: Assessing special children (pp. 18-78). Nueva York: Guilford Press.

36. National Reading Panel. (2000). Teaching children to read: An evidence-based assessment of the scientific research literature on reading and its implications for reading instruction: Reports of the subgroups. Bethesda, MD: National Institute of Child Health and Human Development. Disponible en: http://www.nationalreadingpanel.org/

37. National Research Council (1998). Preventing reading difficulties in young children. Washington, DC: National Academy Press.
38. Shinn, M. R. (Ed.). (1989). Curriculum-based measurement: Assessing special children. Nueva York, NY: Guilford.

39. Smith S. B., Simmons, D. C. y Kame'enui, E. J. (1998). Phonological awareness: Research bases. En D. C. Simmons y E. J. Kame'enui (eds.), What reading research tells us about children with diverse learning needs: Bases and basics. Mahwah, NJ: Lawrence Erlbaum Associates.

40. Shaywitz. S. (2003). Overcoming dyslexia: A new and complete science-based program for reading problems at any level. Nueva York: Knopf.

41. Speece, D.L., Case, L.P. y Molloy, D.E. (2003). The validity of a response-to-instruction paradigm to identify reading disabilities: A longitudinal analysis of individual differences and contextual factors. School Psychology Review, 32, 557-582.

42. Stanovich, K. E. (1986). Matthew effects in reading: Some consequences of individual differences in the acquisition of literacy. Reading Research Quarterly, 21, 360-406. http:// dx.doi.org/10.1598/RRQ.21.4.1

43. Ley IDEA, 2004: http://idea.ed.gov/

44. Vaughn, S. y Fuchs, D. (2003). Redefining learning disabilities as inadequate response to instruction: The promise and potential problems. Learning Disabilities Research \& Practice, 18, 137-146. http://dx.doi.org/10.1111/1540$\underline{5826.00070}$

45. Wallace, T., Espin, C.A., McMaster, K., Deno, S.L. y Foegen, A. (2007). CMB progress monitoring within a standards-based system. The Journal of Special Education, 41, 66-67. http://dx.doi.org/10.1177/00224669070410020201

46. Wanzek, J. y Vaughn, S. (2007). Research-based implications from extensive early reading interventions. School Psychology Review, 36, 541-561.

47. Watson, J. (2004). Examining the reliability and validity of the Indicadores Dinámicos del Exito en la Lectura (IDEL®): A research study. Tesis doctoral, Universidad de Oregon.

Fecha de recepción: 15 de mayo de 2011

Fecha de recepción de la versión modificada: 1 de julio de 2011

Fecha de aceptación: 29 de julio 